\title{
A STUDY OF COMPACT RADIO SOURCES IN NEARBY FACE-ON SPIRAL GALAXIES. II. MULTIWAVELENGTH ANALYSES OF SOURCES IN M51
}

\author{
L. A. Maddox ${ }^{1}$ and J. J. Cowan \\ Homer L. Dodge Department of Physics and Astronomy, University of Oklahoma, Norman, OK 73019, USA \\ R. E. KILGARD \\ Harvard-Smithsonian Center for Astrophysics, Cambridge, MA 02138, USA \\ E. SCHINNERER \\ Max-Planck-Institut für Astronomie, 69117 Heidelberg, Germany \\ AND \\ C. J. Stockdale \\ Department of Physics, Marquette University, Milwaukee, WI 53201, USA \\ Received 2006 October 25; accepted 2007 February 8
}

\begin{abstract}
We report the analysis of deep radio observations of the interacting galaxy system M51 from the Very Large Array, with the goal of understanding the nature of the population of compact radio sources in nearby spiral galaxies. We detect 107 compact radio sources, 64\% of which have optical counterparts in a deep H $\alpha$ Hubble Space Telescope image. Thirteen of the radio sources have X-ray counterparts from a Chandra observation of M51. We find that six of the associated $\mathrm{H} \alpha$ sources are young supernova remnants (SNRs) with resolved shells. Most of the SNRs exhibit steep radio continuum spectral indices consistent with synchrotron emission. We detect emission from the Type Ic SN 1994I nearly a decade after explosion: the emission $\left(160 \pm 22 \mu \mathrm{Jy} \mathrm{beam}^{-1}\right.$ at $20 \mathrm{~cm}, 46 \pm 11 \mu \mathrm{Jy}$ beam ${ }^{-1}$ at $6 \mathrm{~cm}$, $\alpha=-1.02 \pm 0.28$ ) is consistent with light-curve models for Type $\mathrm{Ib} / \mathrm{Ic}$ supernovae. We detect X-ray emission from the supernova; however, no optical counterpart is present. We report on the analysis of the Seyfert 2 nucleus in this galaxy, including the evidence for bipolar outflows from the central black hole.
\end{abstract}

Key words: galaxies: individual (NGC 5194) - H II regions — radio continuum: galaxies supernova remnants $-\mathrm{X}$-rays: galaxies

Online material: machine-readable table

\section{INTRODUCTION}

M51 (NGC 5194/5) is a nearby, grand-design spiral galaxy, with its early-type companion forming an interacting pair. It is nearly face-on $\left(i \sim 20^{\circ}\right)$, which makes it an ideal candidate for population and morphology studies at all wavelengths. This is the second galaxy in our study of nearby spiral galaxies, with the aim of classifying and understanding the populations of compact radio sources in these galaxies. Results of a long-term study of M83 were presented in Paper I of this series (Maddox et al. 2006). Results of our radio observations of M101 and NGC 3184 and a thorough comparison of all four galaxies will be presented in $\mathrm{Pa}-$ per III of this series.

The few distance determinations for M51 range from $6.0 \mathrm{Mpc}$ (Baron et al. 1996) to $9.6 \mathrm{Mpc}$ (Sandage \& Tammann 1974). We have opted to use the distance of $8.4 \mathrm{Mpc}$ as derived by the planetary nebula luminosity function of the galaxy (Feldmeier et al. 1997). Despite the interaction with M51b, the star formation rate (SFR) in the main galaxy M51a is only slightly elevated (Calzetti et al. 2005). Calzetti et al. (2005) report the total SFR as $3.4 M_{\odot} \mathrm{yr}^{-1}$ and the SFR per area as $0.015 M_{\odot} \mathrm{yr}^{-1} \mathrm{kpc}^{-2}$, which place M51a among the "quiescently" star-forming systems. The M51a/b interacting system has played host to three supernovae $(\mathrm{SNe})$ in modern times. SN 1945A occurred in M51b (Kowal \& Sargent 1971), while SN 1994I (Puckett et al. 1994) and SN 2005cs

\footnotetext{
${ }^{1}$ Current address: Department of Astronomy, University of Illinois at UrbanaChampaign, Urbana, IL 61801, USA.
}

(Kloehr et al. 2005) happened in M51a. We report briefly on the radio emission of SN 1994I, the only SN that we have detected in this galaxy with our radio observations. An H I study of M51 by Rots et al. (1990) noted that the neutral gas emission follows the spiral arms closely. The large quantities of gas indicate that compact radio emission should also be concentrated along the arms. M51 contains a low-luminosity Seyfert 2 nucleus that exhibits evidence of a bipolar outflow (e.g., Ford et al. 1985).

In this paper we present multiwavelength analyses of the numerous compact radio sources present in M51 using new, highresolution observations from the Very Large Array (VLA). ${ }^{2}$ Consistent with our previous work on M83, we find that our detected objects fall into three categories. First, thermal $\mathrm{H}$ II regions that are star-forming regions, whose radio emission is dominated by thermal bremsstrahlung, and which have generally flat continuum spectra. At the distances of the sample galaxies, we expect to detect emission only from the strongest $\mathrm{H}$ II emission regions. Second, we detect SNe and supernova remnants (SNRs) that emit radio continuum emission through a shock interaction with the circumstellar material (CSM) or the interstellar medium (ISM), respectively. SNe and SNRs are characterized by a nonthermal continuum spectrum, which fades over timescales of months to years. The final class of objects we detect are X-ray binaries (XRBs) in radio-loud or flaring states. The emission mechanism

\footnotetext{
${ }^{2}$ The Very Large Array of the National Radio Astronomy Observatory is a facility of the National Science Foundation operated in cooperative agreement by Associated Universities, Inc.
} 
TABLE 1

M51 OBSERVATIONS

\begin{tabular}{|c|c|c|c|c|c|}
\hline $\begin{array}{c}\text { Observing Band } \\
(\mathrm{GHz})\end{array}$ & Configuration & Date & $\begin{array}{l}\text { Integration Time } \\
\text { (minutes) }\end{array}$ & $\begin{array}{l}\text { Clean Beam } \\
(\operatorname{arcsec})\end{array}$ & $\begin{array}{c}\text { rms Noise } \\
\left(\mu \mathrm{Jy} \mathrm{beam}^{-1}\right)\end{array}$ \\
\hline $1.425 \ldots \ldots \ldots \ldots$ & A & 2002 Apr 5 & 359.0 & $1.50 \times 1.21$ & 22.5 \\
\hline \multirow[t]{8}{*}{4.860.} & $\mathrm{~B}$ & 2003 Dec 11 & 426.5 & $1.47 \times 1.13$ & 11.7 \\
\hline & & 2003 Dec 12 & 212.5 & & \\
\hline & & 2003 Dec 20 & 90.2 & & \\
\hline & & 2003 Dec 28 & 119.0 & & \\
\hline & & 2003 Dec 29 & 91.5 & & \\
\hline & & 2004 Jan 2 & 120.3 & & \\
\hline & & 2004 Jan 5 & 120.3 & & \\
\hline & & 2004 Jan 9 & 170.5 & & \\
\hline
\end{tabular}

here is the formation of bipolar jets, which interact with the magnetic field of the compact member, producing synchrotron emission. Like the SN/SNR emission, XRB emission is characterized by a steep spectrum in the radio. These sources are also transient in the radio, going through periods of high activity after periods of radio quiescence. In this paper we present details of the radio observations and analysis of M51. We compare our radio observations with archived optical Hubble Space Telescope (HST) and Chandra $\mathrm{X}$-ray data to find counterparts in these complementary bands. Finally, we discuss the radio emission from the Seyfert 2 nucleus.

\section{OBSERVATIONS AND DATA REDUCTION}

Observational parameters for these data are presented in Table 1. The $20 \mathrm{~cm}$ observation was performed on 2002 April 5 for a total of $6 \mathrm{hr}$ while the VLA was in A configuration. The $6 \mathrm{~cm}$ observations were performed in B configuration over several days in 2003 December and 2004 January. The total on-source integration time for the $6 \mathrm{~cm}$ observations was $22.5 \mathrm{hr}$.

All of the data were processed using the Astronomical Image Processing System (AIPS) provided by NRAO. Flux calibration was performed using $3 \mathrm{C} 286$ as the primary calibrator. To correct for atmospheric phase variations, a secondary calibrator, J1327+ 434, was used in all observations. The data were then imaged using the AIPS task IMAGR using a Briggs robustness parameter of 0 . This value has the advantage of minimizing noise while allowing for excellent point-source detection in resulting images. Due to the low flux density in the radio sources and the low dynamic range, self-calibration was not required. The data sets were deconvolved and then restored using Gaussian restoring beams, the dimensions of which are indicated in Table 1 . The size of the Gaussian restoring beams were determined using the values for the "dirty" beam, calculated from the Fourier transform of the $u-v$ plane coverage. After imaging, the task PBCOR was run on each map to correct for the response of the primary beam.

The final $20 \mathrm{~cm}$ radio map achieved a sensitivity of $22.5 \mu \mathrm{Jy}$ beam ${ }^{-1} \mathrm{rms}$ with a deconvolved beam measuring $1.50^{\prime \prime} \times 1.21^{\prime \prime}$. The $6 \mathrm{~cm}$ map reached $11.7 \mu \mathrm{Jy}_{\text {beam }}{ }^{-1} \mathrm{rms}$ with a deconvolved beam of $1.47^{\prime \prime} \times 1.13^{\prime \prime}$. Each map used a pixel scale of $0.3^{\prime \prime}$ pixel $^{-1}$ and covers a sky area of $10.2^{\prime}$ per side.

\section{DATA ANALYSIS}

An initial source list was obtained using the AIPS task SAD, which searches for points in a radio map that are higher than a specified level. Our search consisted of four iterations, each looking at fainter flux levels. The flux cutoff levels were 1.0, 0.5, 0.1, and $0.05 \mathrm{mJy} \mathrm{beam}^{-1}$. A Gaussian fit was applied to each detected emission source by the program, and fits that fail are rejected by the algorithm. Some extended or slightly extended sources were listed as multiple entries. Sources that exhibited extended emission, larger than 2 beamwidths across $\left(>2.5^{\prime \prime}\right)$, were removed. Our source list was compressed to account for this. Finally, a visual inspection of the maps was performed in order to find real emission that was rejected by the detection algorithm. This was the case for 10 faint sources that were detected in both wavelengths at identical positions. The final list of sources, including peak flux densities and spectral indices $\left(S \propto \nu^{+\alpha}\right)$, is listed in Table 2. The final $20 \mathrm{~cm}$ radio map is shown in Figure 1. The spiral arms are evident in the radio maps, as is the position of M51b, the interacting companion galaxy. As a comparison, Figure 2 shows a threecolor HST image of M51.

The peak flux density and rms values on the final source list were determined using the AIPS task IMFIT. The input model was a simple two-dimensional Gaussian. Peak flux densities are listed in Table 2. Where the fit failed, we assume a $3 \sigma$ upper limit of $65 \mu \mathrm{Jy}$ at $20 \mathrm{~cm}$ and $36 \mu \mathrm{Jy}$ at $6 \mathrm{~cm}$.

\section{DISCUSSION}

\subsection{Radio Point Sources}

In Table 2 we present the results of our radio survey of the M51 system listing the positions, radio flux densities at 20 and $6 \mathrm{~cm}$ (when detected at the appropriate band), and the spectral index $\left(\alpha, S_{\nu} \propto \nu^{+\alpha}\right)$ derived from these measurements for our 107 radio point sources. We have determined that 33 of these sources have steep indices $(\alpha<-0.3), 45$ sources have flat indices $(-0.3<\alpha<0.3)$, and 3 sources have inverted spectral indices $(0.3<\alpha)$. The final 26 sources cannot be distinguished as having either a flat or steep spectral index due to experimental uncertainties. Figure 3 shows the sources overlaid on an HST ACS I-band image of M51.

Sources $15,20,35,40$, and 78 have very steep spectral indices $(\alpha<-1)$, with sources 40 and 78 having only been detected in the $20 \mathrm{~cm}$ observations. These sources may be background sources (e.g., unresolved radio galaxies), optically obscured SNRs, or highly variable, unresolved radio sources in M51, as the 20 and $6 \mathrm{~cm}$ data were obtained 21 months apart. Source 20 is associated with an optical $\mathrm{H}$ II region, so it is likely this source is an optically obscured SNR.

All of the detected radio sources lie along the spiral arms of the galaxy. The three inverted sources, denoted as blue circles in Figure 3, lie in dust lanes in the inner region of the galaxy. They could be embedded $\mathrm{H}$ II regions experiencing free-free absorption due to a high column density of gas. In order to test for free-free absorption in these compact sources, we would require highresolution radio observations at lower and higher frequencies to look for a spectral turnover at low frequency. A similar study was performed by Tingay (2004) for NGC 253, who found significant 
TABLE 2

Positions, Flux Densities, and Spectral Indices of Point Sources in M51

\begin{tabular}{|c|c|c|c|c|c|}
\hline Source & R.A. (J2000.0) & Decl. (J2000.0) & $\begin{array}{c}20 \mathrm{~cm} \\
\left(\mu \mathrm{Jy} \mathrm{beam}^{-1}\right)\end{array}$ & $\begin{array}{c}6 \mathrm{~cm} \\
\left(\mu \mathrm{Jy} \mathrm{beam}^{-1}\right)\end{array}$ & Spectral Index $\alpha^{\mathrm{a}}$ \\
\hline $1 \ldots \ldots$ & 132930.46 & 471250.61 & $394 \pm 21$ & $190 \pm 18$ & $-0.59 \pm 0.11$ \\
\hline ................ & 132936.56 & 471105.51 & $68 \pm 21$ & $43 \pm 13$ & $-0.37 \pm 0.43$ \\
\hline 3 & 132938.05 & 471205.84 & $470 \pm 22$ & $264 \pm 14$ & $-0.47 \pm 0.07$ \\
\hline 4 & 132938.96 & 471323.60 & $84 \pm 22$ & $102 \pm 18$ & $+0.15 \pm 0.32$ \\
\hline $5^{\mathrm{b}}$ & 132939.36 & 470840.72 & $\ldots$ & $73 \pm 17$ & $>+0.09$ \\
\hline $6^{\mathrm{b}}$ & 132943.67 & 471000.95 & $56 \pm 21$ & $32 \pm 13$ & $-0.46 \pm 0.55$ \\
\hline 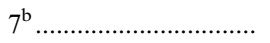 & 132944.05 & 471022.71 & $245 \pm 20$ & $259 \pm 12$ & $+0.05 \pm 0.09$ \\
\hline ……............ & 132945.10 & 471332.25 & $164 \pm 21$ & $93 \pm 13$ & $-0.46 \pm 0.19$ \\
\hline $9^{\mathrm{b}}$ & 132945.17 & 470956.93 & $192 \pm 21$ & $229 \pm 12$ & $+0.14 \pm 0.12$ \\
\hline $10^{\mathrm{b}}$ & 132946.40 & 471233.14 & $55 \pm 22$ & $75 \pm 12$ & $+0.25 \pm 0.43$ \\
\hline $11^{\mathrm{b}}$ & 132946.74 & 470940.78 & $\ldots$ & $61 \pm 13$ & $>-0.06$ \\
\hline $12 \ldots \ldots \ldots$ & 132949.14 & 471257.11 & $123 \pm 21$ & $43 \pm 15$ & $-0.86 \pm 0.27$ \\
\hline $13^{\mathrm{b}}$ & 132949.43 & 471240.54 & $\ldots$ & $217 \pm 11$ & $>+0.98$ \\
\hline 14 & 132949.53 & 471400.17 & $234 \pm 21$ & $221 \pm 13$ & $-0.05 \pm 0.11$ \\
\hline $15 \ldots \ldots$ & 132949.60 & 471327.51 & $326 \pm 21$ & $74 \pm 12$ & $-1.21 \pm 0.17$ \\
\hline 16 & 132949.43 & 471123.86 & $123 \pm 20$ & $49 \pm 17$ & $-0.75 \pm 0.38$ \\
\hline 17 & 132949.93 & 471120.53 & $121 \pm 19$ & $232 \pm 11$ & $+0.53 \pm 0.16$ \\
\hline 18 & 132949.93 & 471131.11 & $464 \pm 22$ & $235 \pm 11$ & $-0.55 \pm 0.07$ \\
\hline $19^{\mathrm{b}} \ldots \ldots \ldots \ldots \ldots \ldots \ldots \ldots \ldots \ldots$ & 132949.95 & 471126.73 & $234 \pm 21$ & $113 \pm 10$ & $-0.59 \pm 0.13$ \\
\hline$\ldots \ldots \ldots \ldots . .$. & 132950.04 & 471124.89 & $205 \pm 21$ & $43 \pm 17$ & $-1.20 \pm 0.41$ \\
\hline $21 \ldots \ldots \ldots$ & 132950.13 & 471140.32 & $140 \pm 20$ & $54 \pm 10$ & $-0.78 \pm 0.23$ \\
\hline $22^{\mathrm{b}} \ldots \ldots \ldots \ldots \ldots \ldots \ldots \ldots \ldots \ldots$ & 132950.13 & 471136.92 & $107 \pm 20$ & $59 \pm 10$ & $-0.49 \pm 0.25$ \\
\hline $23 \ldots \ldots \ldots \ldots \ldots \ldots$ & 132950.20 & 471151.36 & $146 \pm 20$ & $103 \pm 11$ & $-0.28 \pm 0.17$ \\
\hline $24^{\mathrm{b}}$ & 132950.24 & $4711 \quad 19.06$ & $71 \pm 21$ & $63 \pm 11$ & $-0.10 \pm 0.34$ \\
\hline $25 \ldots \ldots \ldots \ldots \ldots \ldots \ldots$ & 132950.26 & 471148.48 & $\ldots$ & $54 \pm 10$ & $>-0.16$ \\
\hline $26 \ldots \ldots \ldots \ldots \ldots \ldots$ & 132950.30 & 471122.42 & $432 \pm 21$ & $240 \pm 11$ & $-0.48 \pm 0.07$ \\
\hline $27 \ldots \ldots \ldots \ldots \ldots \ldots \ldots$ & 132950.32 & 471357.95 & $61 \pm 20$ & $63 \pm 13$ & $+0.026 \pm 0.87$ \\
\hline $28 \ldots \ldots \ldots \ldots \ldots \ldots \ldots$ & 132950.32 & 471132.91 & $111 \pm 17$ & $63 \pm 8$ & $-0.46 \pm 0.20$ \\
\hline $29 \ldots \ldots \ldots \ldots$ & 132950.43 & 471153.26 & $\ldots$ & $70 \pm 10$ & $>0.06$ \\
\hline $30 \ldots \ldots \ldots$ & 132950.45 & 471145.16 & $56 \pm 20$ & $55 \pm 11$ & $-0.01 \pm 0.41$ \\
\hline $31 \ldots \ldots \ldots \ldots \ldots \ldots \ldots$ & 132950.45 & 471127.02 & $115 \pm 20$ & $73 \pm 10$ & $-0.37 \pm 0.22$ \\
\hline $32 .$. & 132950.46 & 471137.36 & $\ldots$ & $59 \pm 9$ & $>-0.08$ \\
\hline 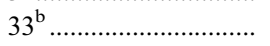 & 132950.48 & $47 \quad 1345.22$ & $82 \pm 21$ & $73 \pm 12$ & $-0.09 \pm 0.3$ \\
\hline $34 \ldots \ldots \ldots \ldots \ldots \ldots \ldots$ & 132950.70 & 471155.86 & $\ldots$ & $122 \pm 10$ & $>+0.51$ \\
\hline 35 & 132950.95 & 471343.69 & $\ldots$ & $84 \pm 12$ & $>+0.20$ \\
\hline 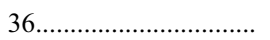 & 132951.50 & 471200.54 & $213 \pm 21$ & $165 \pm 11$ & $-0.21 \pm 0.12$ \\
\hline $37 \ldots \ldots \ldots$ & 132951.57 & 471208.01 & $2229 \pm 21$ & $946 \pm 11$ & $-0.70 \pm 0.015$ \\
\hline $38 \ldots \ldots \ldots \ldots \ldots \ldots \ldots$ & 132951.68 & 471157.89 & $52 \pm 20$ & $49 \pm 10$ & $-0.05 \pm 0.44$ \\
\hline 39 & 132951.73 & 471201.87 & $108 \pm 21$ & $132 \pm 11$ & $+0.16 \pm 0.21$ \\
\hline $40 \ldots \ldots \ldots \ldots \ldots \ldots$ & 132951.80 & 471140.43 & $385 \pm 20$ & $\ldots$ & $<-1.99$ \\
\hline 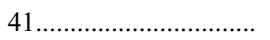 & 132951.86 & 471137.01 & $211 \pm 20$ & $115 \pm 10$ & $-0.49 \pm 0.13$ \\
\hline $42^{\mathrm{b}}$ & 132951.99 & 471054.02 & $115 \pm 20$ & $52 \pm 11$ & $-0.65 \pm 0.27$ \\
\hline 43 & 132952.01 & $47 \quad 1202.21$ & $\ldots$ & $55 \pm 10$ & $>-0.14$ \\
\hline $44^{\mathrm{b}}$ & 132952.01 & 471242.95 & $151 \pm 21$ & $173 \pm 11$ & $+0.11 \pm 0.15$ \\
\hline 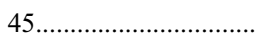 & 132952.02 & $47 \quad 1204.42$ & $\ldots$ & $57 \pm 10$ & $>-0.11$ \\
\hline 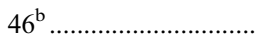 & 132952.03 & $47 \quad 1247.20$ & $64 \pm 20$ & $62 \pm 11$ & $-0.03 \pm 0.36$ \\
\hline $47^{\mathrm{b}}$ & 132952.08 & 471126.82 & $84 \pm 21$ & $40 \pm 13$ & $-0.60 \pm 0.41$ \\
\hline 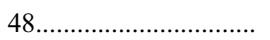 & 132952.17 & 471136.60 & $94 \pm 20$ & $73 \pm 10$ & $-0.21 \pm 0.25$ \\
\hline $49^{\mathrm{b}}$ & 132952.22 & 471129.48 & $87 \pm 29$ & $50 \pm 13$ & $-0.45 \pm 0.42$ \\
\hline 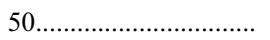 & 132952.35 & 471136.09 & $94 \pm 20$ & $59 \pm 10$ & $-0.38 \pm 0.27$ \\
\hline $51^{\mathrm{b}}$ & 132952.38 & 471238.51 & $\ldots$ & $56 \pm 11$ & $>-0.13$ \\
\hline $52^{\mathrm{b}}$ & 132952.73 & $\begin{array}{lll}47 & 11 & 21.23\end{array}$ & $162 \pm 26$ & $59 \pm 13$ & $-0.82 \pm 0.27$ \\
\hline 53 & 132952.71 & 471142.73 & $2079 \pm 21$ & $1135 \pm 11$ & $-0.49 \pm 0.01$ \\
\hline 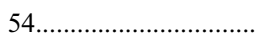 & 132952.79 & 471139.17 & $2047 \pm 20$ & $761 \pm 10$ & $-0.81 \pm 0.02$ \\
\hline $55^{\mathrm{b}}$ & 132953.22 & 471239.53 & $94 \pm 21$ & $65 \pm 11$ & $-0.30 \pm 0.28$ \\
\hline $56 \ldots \ldots \ldots \ldots \ldots \ldots \ldots \ldots \ldots \ldots \ldots$ & 132953.86 & 470954.09 & $152 \pm 22$ & $73 \pm 12$ & $-0.60 \pm 0.22$ \\
\hline 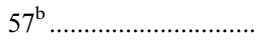 & 132953.91 & 471405.44 & $\ldots$ & $61 \pm 13$ & $>-0.06$ \\
\hline 58 & 132954.12 & 471130.33 & $160 \pm 22$ & $46 \pm 11$ & $-1.02 \pm 0.28$ \\
\hline 59 & 132954.24 & 471132.40 & $136 \pm 22$ & $55 \pm 11$ & $-0.74 \pm 0.26$ \\
\hline $60 \ldots \ldots \ldots \ldots \ldots \ldots \ldots \ldots$ & 132954.24 & 471123.23 & $81 \pm 21$ & $45 \pm 11$ & $-0.48 \pm 0.36$ \\
\hline 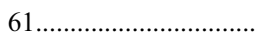 & 132954.32 & 471129.86 & $92 \pm 21$ & $67 \pm 11$ & $-0.26 \pm 0.28$ \\
\hline 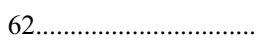 & 132954.72 & 471236.60 & $98 \pm 22$ & $49 \pm 11$ & $-0.56 \pm 0.32$ \\
\hline 63............................... & 132954.84 & 471159.23 & $51 \pm 19$ & $36 \pm 10$ & $-0.28 \pm 0.46$ \\
\hline $64^{\mathrm{b}}$ & 132954.92 & 471133.00 & $133 \pm 20$ & $72 \pm 10$ & $-0.50 \pm 0.20$ \\
\hline 65 & 132954.95 & 470922.41 & $406 \pm 21$ & $280 \pm 17$ & $-0.30 \pm 0.08$ \\
\hline
\end{tabular}


TABLE 2-Continued

\begin{tabular}{|c|c|c|c|c|c|}
\hline Source & R.A. (J2000.0) & Decl. (J2000.0) & $\begin{array}{c}20 \mathrm{~cm} \\
\left(\mu \mathrm{Jy} \mathrm{beam}^{-1}\right)\end{array}$ & $\begin{array}{c}6 \mathrm{~cm} \\
\left(\mu \mathrm{Jy} \mathrm{beam}^{-1}\right)\end{array}$ & Spectral Index $\alpha^{\mathrm{a}}$ \\
\hline 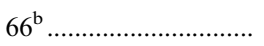 & 132954.98 & 471048.93 & $60 \pm 21$ & $62 \pm 11$ & $+0.03 \pm 0.39$ \\
\hline $67^{\mathrm{b}}$ & 132955.08 & 471135.01 & $126 \pm 20$ & $89 \pm 10$ & $-0.28 \pm 0.19$ \\
\hline $68^{\mathrm{b}}$ & 132955.25 & 471046.16 & $158 \pm 23$ & $61 \pm 14$ & $-0.78 \pm 0.27$ \\
\hline 69. & 132955.31 & 471138.61 & $133 \pm 20$ & $54 \pm 10$ & $-0.73 \pm 0.24$ \\
\hline 70 & 132955.33 & 471136.51 & $\ldots$ & $59 \pm 10$ & $>-0.08$ \\
\hline $71 \ldots$ & 132955.33 & $47 \quad 1202.32$ & $\ldots$ & $44 \pm 10$ & $>-0.32$ \\
\hline $72 \ldots \ldots \ldots \ldots \ldots \ldots \ldots \ldots \ldots$ & 132955.42 & 471140.67 & $\ldots$ & $51 \pm 10$ & $>-0.20$ \\
\hline $73^{\mathrm{b}}$ & 132955.42 & 471402.05 & $101 \pm 23$ & $80 \pm 16$ & $-0.19 \pm 0.30$ \\
\hline 74 & 132955.52 & 471209.92 & $98 \pm 20$ & $54 \pm 10$ & $-0.49 \pm 0.28$ \\
\hline 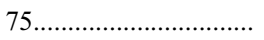 & 132955.57 & 471359.82 & $175 \pm 15$ & $78 \pm 12$ & $-0.66 \pm 0.18$ \\
\hline 76 & 132955.60 & 471203.08 & $80 \pm 20$ & $37 \pm 10$ & $-0.63 \pm 0.37$ \\
\hline 77 & 132955.64 & 471141.57 & $\ldots$ & $53 \pm 10$ & $>-0.17$ \\
\hline 78. & 132955.69 & 471146.61 & $122 \pm 18$ & $\ldots$ & $<-1.04$ \\
\hline $79^{\mathrm{b}}$ & 132955.86 & 471144.48 & $319 \pm 25$ & $230 \pm 13$ & $-0.26 \pm 0.10$ \\
\hline 80 & 132955.85 & 471154.58 & $246 \pm 22$ & $142 \pm 11$ & $-0.45 \pm 0.12$ \\
\hline $81 \ldots \ldots \ldots \ldots$ & 132955.86 & 471150.61 & $147 \pm 21$ & $61 \pm 11$ & $-0.72 \pm 0.23$ \\
\hline $82^{\mathrm{b}}$ & 132956.13 & 471408.91 & $77 \pm 22$ & $78 \pm 14$ & $+0.01 \pm 0.34$ \\
\hline 83 & 132956.21 & 471047.33 & $109 \pm 21$ & $73 \pm 11$ & $-0.33 \pm 0.24$ \\
\hline 84 & 132957.47 & 471037.08 & $128 \pm 22$ & $59 \pm 14$ & $-0.63 \pm 0.29$ \\
\hline $85^{\mathrm{b}}$ & 132958.94 & 471409.00 & $98 \pm 22$ & $89 \pm 13$ & $-0.08 \pm 0.27$ \\
\hline 86 & 132959.49 & 471109.96 & $\ldots$ & $68 \pm 11$ & $>+0.03$ \\
\hline $87^{\mathrm{b}}$ & 132959.53 & 471558.30 & $1494 \pm 20$ & $1181 \pm 21$ & $-0.19 \pm 0.02$ \\
\hline 88 & 132959.58 & $4711 \quad 11.32$ & $95 \pm 20$ & $77 \pm 11$ & $-0.17 \pm 0.25$ \\
\hline $89^{\mathrm{b}}$ & 132959.61 & $47 \quad 1359.18$ & $88 \pm 20$ & $84 \pm 13$ & $-0.04 \pm 0.27$ \\
\hline $90 \ldots \ldots \ldots \ldots$ & 132959.84 & 471112.68 & $238 \pm 21$ & $307 \pm 12$ & $+0.21 \pm 0.10$ \\
\hline $91^{\mathrm{b}}$. & 133000.11 & 471330.53 & $63 \pm 21$ & $66 \pm 13$ & $+0.04 \pm 0.39$ \\
\hline $92^{\mathrm{b}}$. & 133000.36 & 471318.87 & $82 \pm 21$ & $76 \pm 13$ & $-0.06 \pm 0.31$ \\
\hline $93^{\mathrm{b}}$ & 133000.78 & 471137.73 & $\ldots$ & $58 \pm 11$ & $>-0.10$ \\
\hline $94^{\mathrm{b}}$ & 133000.93 & 470928.90 & $61 \pm 18$ & $73 \pm 13$ & $+0.15 \pm 0.34$ \\
\hline 95 & 133001.27 & 471243.77 & $131 \pm 22$ & $62 \pm 15$ & $-0.61 \pm 0.29$ \\
\hline 96 & 133001.41 & 471157.83 & $78 \pm 20$ & $50 \pm 11$ & $-0.36 \pm 0.34$ \\
\hline 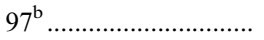 & 133001.50 & 471251.42 & $412 \pm 21$ & $443 \pm 12$ & $+0.06 \pm 0.06$ \\
\hline $98^{\mathrm{b}}$ & 133001.77 & 471148.81 & $54 \pm 22$ & $60 \pm 12$ & $+0.09 \pm 0.45$ \\
\hline 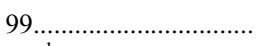 & 133002.03 & 470951.35 & $113 \pm 21$ & $75 \pm 13$ & $-0.33 \pm 0.25$ \\
\hline $100^{\mathrm{b}} \ldots \ldots \ldots \ldots \ldots \ldots \ldots$ & 133002.38 & 470949.10 & $170 \pm 21$ & $194 \pm 13$ & $+0.11 \pm 0.14$ \\
\hline 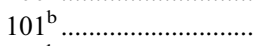 & 133002.75 & 470956.94 & $67 \pm 21$ & $64 \pm 13$ & $-0.04 \pm 0.37$ \\
\hline $102^{\mathrm{b}}$ & 133003.50 & 470941.00 & $\ldots$ & $78 \pm 14$ & $>+0.14$ \\
\hline 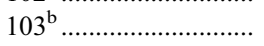 & 133003.95 & 471533.00 & $119 \pm 21$ & $144 \pm 21$ & $+0.16 \pm 0.23$ \\
\hline $104 \ldots \ldots \ldots \ldots$ & 133005.13 & 471035.78 & $9599 \pm 22$ & $4287 \pm 14$ & $-0.66 \pm 0.00$ \\
\hline $105^{\mathrm{b}} \ldots \ldots \ldots \ldots \ldots \ldots \ldots \ldots$ & 133007.38 & 471322.30 & $144 \pm 21$ & $175 \pm 15$ & $+0.16 \pm 0.17$ \\
\hline $106 \ldots \ldots \ldots \ldots \ldots$ & 133010.85 & 470940.26 & $148 \pm 21$ & $100 \pm 17$ & $-0.32 \pm 0.22$ \\
\hline $107 \ldots \ldots \ldots \ldots$ & 133011.03 & 471040.75 & $500 \pm 22$ & $482 \pm 20$ & $-0.03 \pm 0.06$ \\
\hline
\end{tabular}

Notes.-Table 2 is also available in machine-readable form in the electronic edition of the Astronomical Journal. Units of right ascension are hours, minutes, and seconds, and units of declination are degrees, arcminutes, and arcseconds.

${ }^{\mathrm{a}} S_{\nu} \propto \nu^{+\alpha}$.

${ }^{\mathrm{b}}$ Coincident with the optical $\mathrm{H}$ II region.

evidence for free-free absorption of the compact radio emission regions.

The flat spectrum sources in Figure 3, indicated in yellow, tend to populate the outer spiral arms, away from dust-rich areas. The steep-spectrum sources are seen mostly along the inner arms. If these sources are radio SNRs, the position along the inner region is not unusual, as star formation in this region would be higher. The indeterminant sources also lie mostly in the inner region. Some of these sources might be absorbed SNRs, as they are positioned along the dust lanes. High-resolution, narrowband optical observations (e.g., [O III] and [S II]) would be required to further classify these objects.

We have identified 44 radio sources coincident with previously detected optical $\mathrm{H}$ II regions. Of these, 27 sources have flat spectral indices, 8 have steep spectral indices, and 8 have indices that cannot be classified as either due to experimental uncertainty.
One of the 45 sources, source 13, has an inverted spectral index $>1.0$. This source is likely embedded in the disk, with the $20 \mathrm{~cm}$ flux being absorbed by intervening gas (e.g., Tingay 2004). Sources 17 and 34 , with spectral indices of $\sim 0.5$, are also likely embedded $\mathrm{H}$ II regions.

\subsubsection{Supernovae}

At the time of our observations (2002 April and 2004 January), M51 had been the host to two historical SNe: the Type I SN 1945A (Kowal \& Sargent 1971), and the Type Ic SN1994I (Puckett et al. 1994). We were unable to detect emission from the reported position of SN 1945A, which exploded in M51b and would be near the edge of our field of view. Figure 2 shows the position of the SNe on an optical HST image of M51.

We have detected nonthermal radio emission from the reported optical position of SN 1994I (source 58; Puckett et al. 1994), 


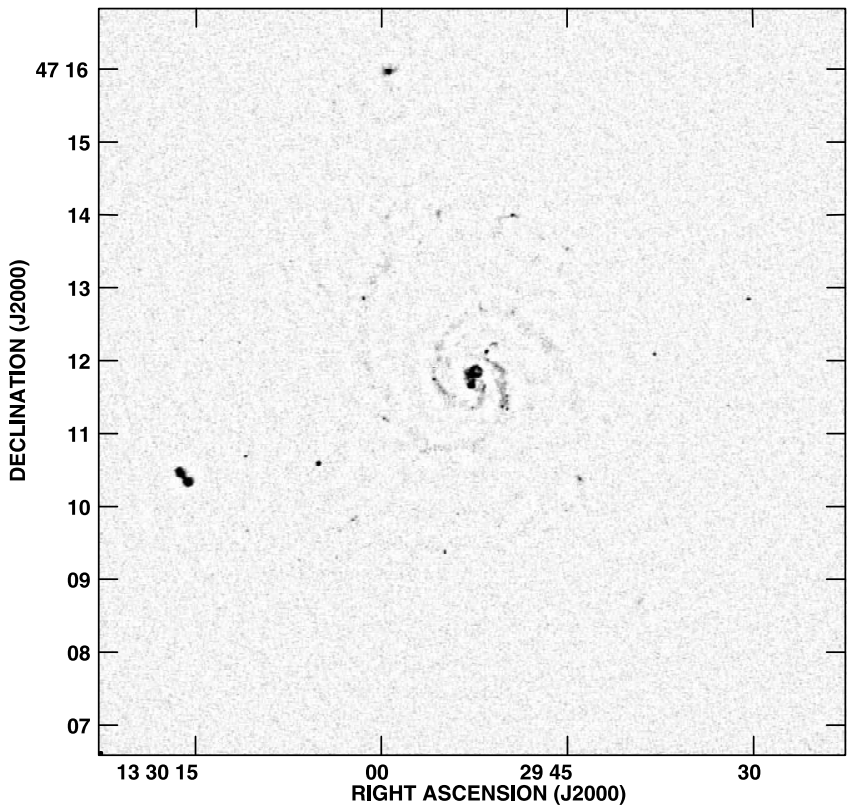

FIG. 1.-The $20 \mathrm{~cm}$ VLA radio map of M51 made in the A configuration with a deconvolved beam of $1.47^{\prime \prime} \times 1.13^{\prime \prime}$. The gray-scale limits are -1 to $500 \mu \mathrm{Jy}$. We note the bright double-lobed radio galaxy east-southeast of the M51 system, which lies outside the plane of the galaxy and is not discussed further.

nearly a decade after its initial discovery (see Fig. 4). The flux level at this time $(160 \mu \mathrm{Jy}$ at $20 \mathrm{~cm})$ is consistent with a Type Ic SN, based on the model of Weiler et al. (2002). If we assume a uniform progenitor wind velocity of $10 \mathrm{~km} \mathrm{~s}^{-1}$, we determine a progenitor mass-loss rate of $\dot{M}=2.75 \times 10^{-6} M_{\odot} \mathrm{yr}^{-1}$

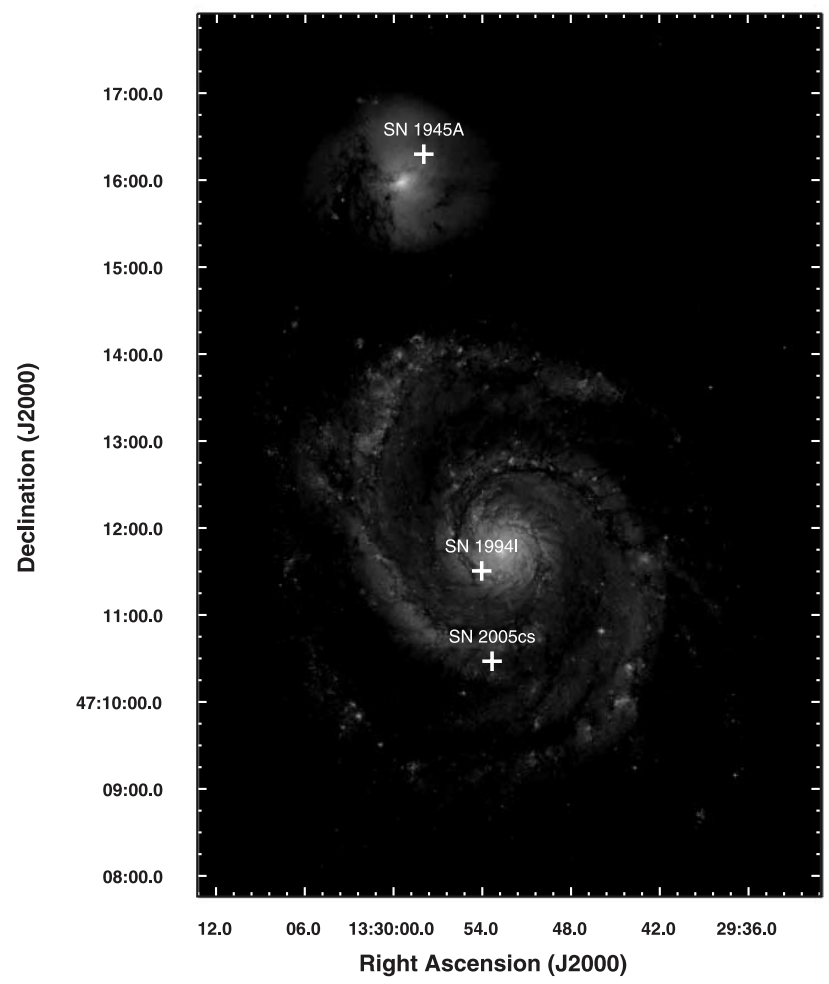

FIG. 2.-Three-color optical image of M51 from HST ACS. The crosses indicate the positions of the historical SNe in the galaxy. Image credit: NASA, ESA, S. Beckwith, and The Hubble Heritage Team (STScI/AURA).

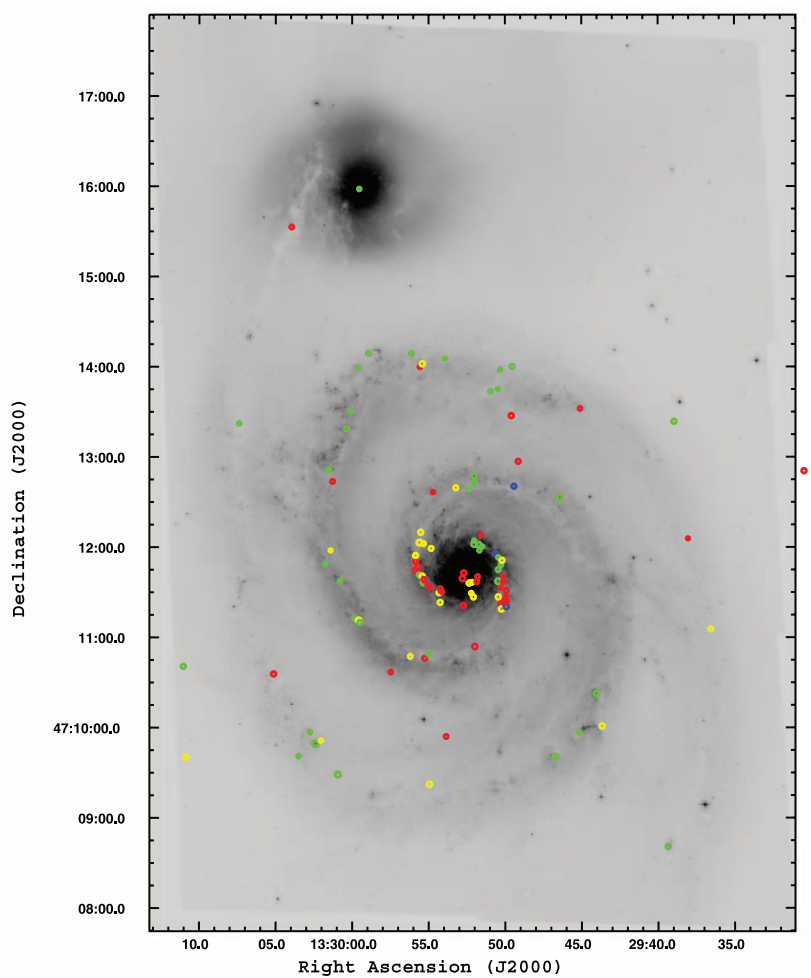

FIG. 3.- I-band image of M51 with radio sources overlaid. We have opted to use the smoother $I$-band image instead of the $\mathrm{H} \alpha$ image as a background, to illustrate the position of the radio sources with respect to the optical galaxy. The radio sources have been binned according to their spectral index: steep spectrum sources in red, flat spectrum sources in green, inverted spectrum sources in blue, and indeterminate flat/steep spectrum sources in yellow.

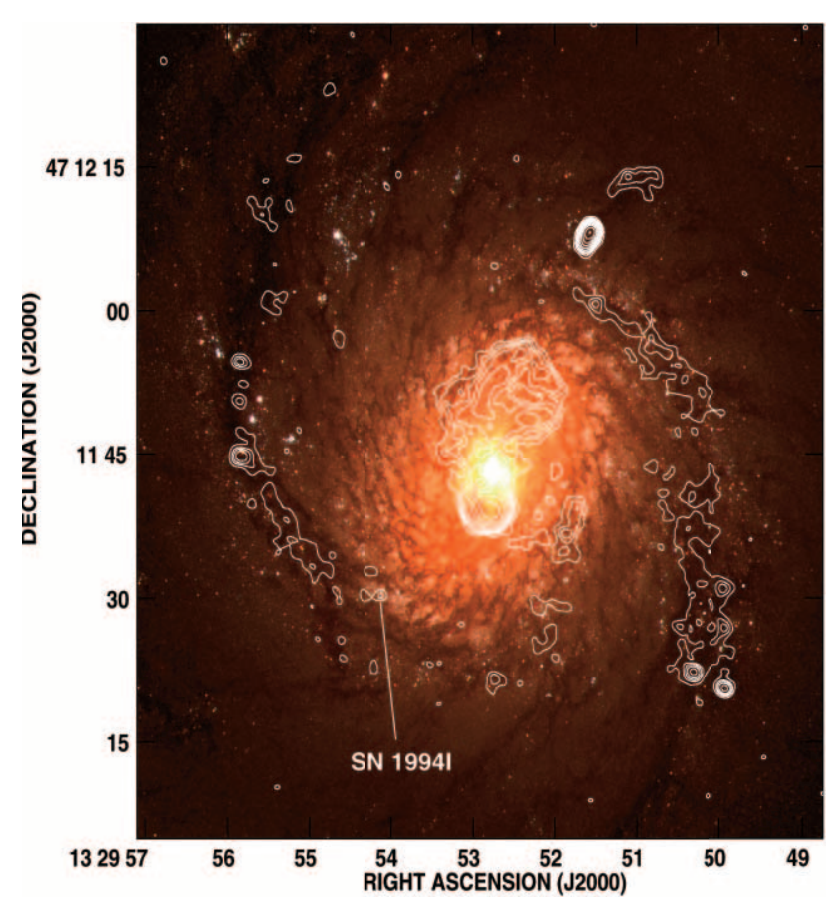

FIG. 4.-Radio contours of $20 \mathrm{~cm}$ emission overlaid on an optical HST image of the central $3.7 \mathrm{kpc}$ of M51a. The position of SN 1994I is marked. VLA observations are made in the $\mathrm{B}$ configuration, with a deconvolved beam of $1.47^{\prime \prime} \times 1.13^{\prime \prime}$. The contour levels are 70, 98, 200, 280, 400, 560, 800, 1120,

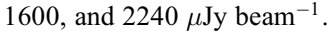



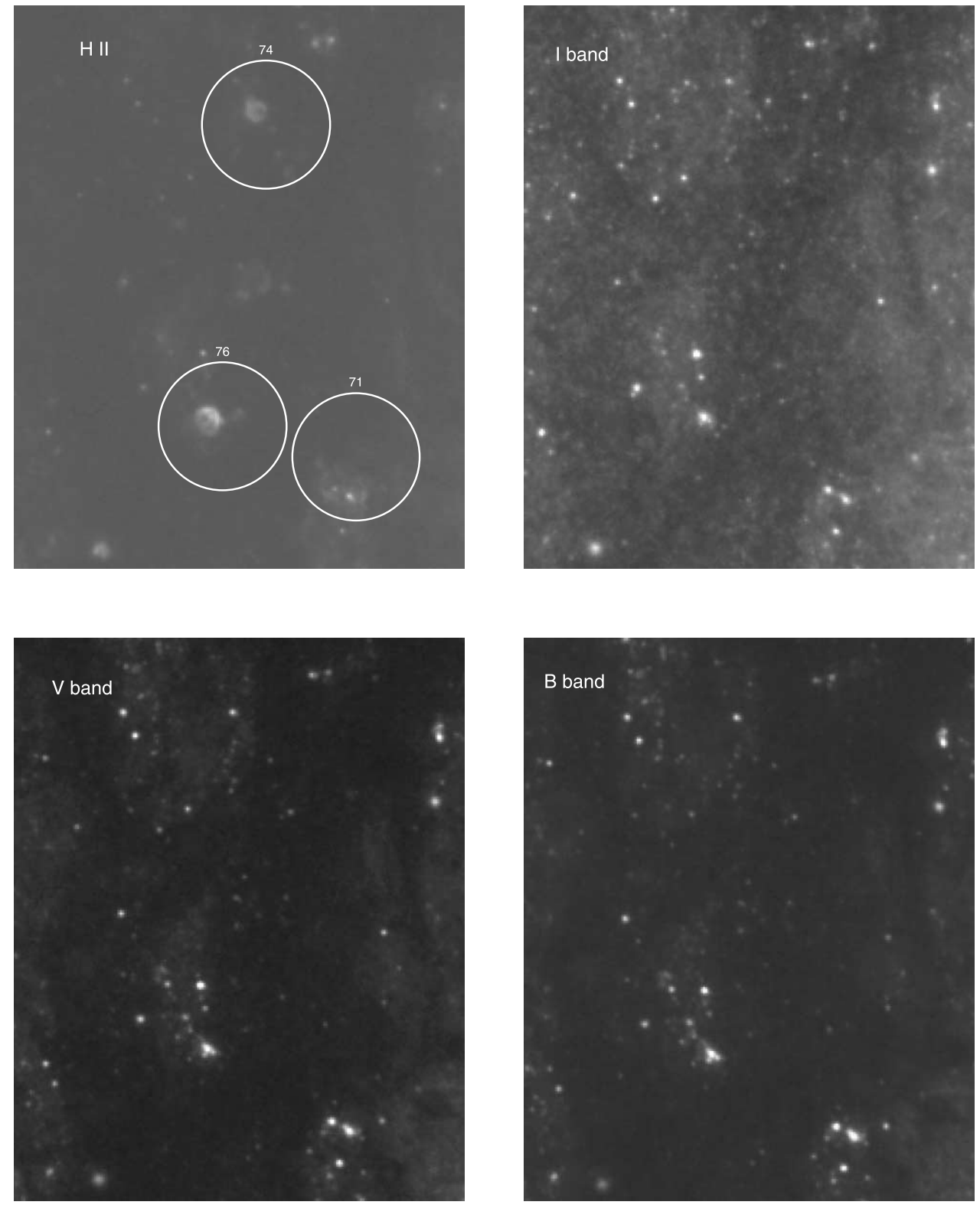

FIG. 5.-Blowup of a small area within M51. The $\mathrm{H} \alpha$ image shows the positions of radio sources, indicated by circles, associated with optical emission. Two of the H $\alpha$ sources indicate shell structure indicative of SNRs. The remaining images show no shell emission. The emission in the other optical bands is predominantly due to starlight.

(Stockdale et al. 2005b). An estimate of the progenitor massloss based on the X-ray emission (using the same wind velocity) yields a rate of $\dot{M} \sim 10^{-5} M_{\odot} \mathrm{yr}^{-1}$, with a reported value for the luminosity of $\sim 10^{37} \mathrm{ergs} \mathrm{s}^{-1}$ (Immler et al. 2002). Our measured radio spectral index for SN 1994I of -1.04 is typical for Type Ic SNe and equivalent with the value reported by Weiler et al. (2002) for the early radio observations of -1.16 . Ten years after explosion, the $6 \mathrm{~cm}$ flux ( $46 \pm 11 \mu \mathrm{Jy})$ is beginning to fade below the detectable limits of a typical deep VLA search.

While our observations predate the explosion of the Type II SN $2005 \mathrm{cs}$, subsequent radio observations of this SN did not detect emission from this source at 1.3, 2.0, 3.5, and $6.2 \mathrm{~cm}$ (Stockdale et al. 2005a). We detect no identifiable radio emission from the immediate nearby region, which would indicate the presence of radio SNRs or H II regions.

\subsubsection{Optical Counterparts}

We overlaid our radio positions on an optical HST ACS image available through the Hubble Heritage project (Fig. 3). Of our 107 compact radio sources, 44 are coincident with large $\mathrm{H}$ II regions detected in $\mathrm{H} \alpha$. An additional 24 have more compact $\mathrm{H} \alpha$ counterparts associated with stellar clusters. Six of these compact sources $(5,47,68,74,76$, and 84$)$ are coincident with resolved $\mathrm{H} \alpha$ shells ( Fig. 5). Other broad optical bands show only emission from stars at these positions. Two of the sources, 47 and 84 , have associated X-ray emission, and are discussed in $\S$ 4.1.3. The remaining sources, with the exception of source 5, exhibit nonthermal radio emission consistent with that of SNRs. The angular diameters of most remnants, as seen in the $\mathrm{H} \alpha$ images, are $\sim 0.3^{\prime \prime}-0.5^{\prime \prime}$, corresponding to $12.2-20.4 \mathrm{pc}$. If we assume a Cas Alike expansion velocity of $6000 \mathrm{~km} \mathrm{~s}^{-1}$, this would give an age of $\sim 2000-3300 \mathrm{yr}$ for the largest shell source.

The remaining $\mathrm{H}$ II counterparts lie in large clouds of ionized gas. In other optical filters, these clouds contain large star clusters (Fig. 6). Most of these radio sources have flat spectral emission, but a few exhibit nonthermal spectra similar to the emission from the shell sources. Four of the sources in this category have X-ray counterparts, and are discussed in $\S$ 4.1.3. 

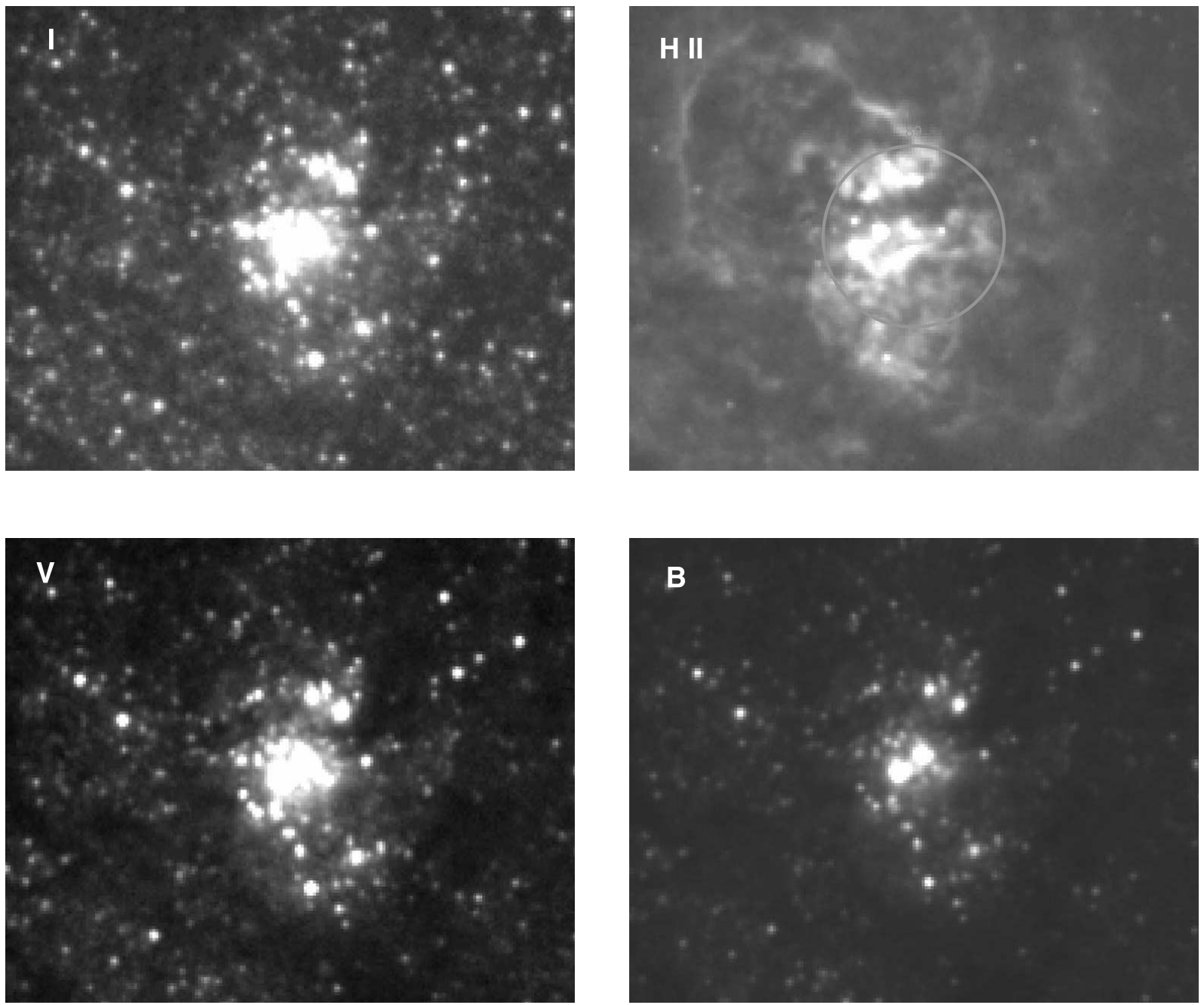

FIG. 6.-Source 89. Many sources, such as this one, lie in dense stellar clusters. Source 89 (circled in the H $\alpha$ image) is a flat-spectrum radio source that is coincident with a large $\mathrm{H}$ II region. In the $I, V$, and $B$ bands it is quite evident that the photoionization of the $\mathrm{H} \alpha$ cloud is powered by the stars in the cluster.

To estimate the strength of the $\mathrm{H}$ II regions in M51, we have computed excitation parameters based on the $6 \mathrm{~cm}$ radio properties that we have determined from our observations. Using the formulae from Schraml \& Mezger (1969) and Mezger \& Henderson (1967) we calculate the excitation parameter $U$ :

$$
U=4.5526\left[a(\nu, T)^{-1} \nu^{0.1} T_{e}^{0.35} S_{\nu} D^{2}\right]^{1 / 3}
$$

where

$$
\begin{aligned}
a(\nu, T)= & 0.366\left(\frac{\nu}{\mathrm{GHz}}\right)^{0.1}\left(\frac{T_{e}}{\mathrm{~K}}\right)^{-0.15} \\
& \times\left\{\ln \left[4.995 \times 10^{-2}\left(\frac{\nu}{\mathrm{GHz}}\right)^{-1}\right]+1.5 \ln \left(\frac{T_{e}}{\mathrm{~K}}\right)\right\},
\end{aligned}
$$

and a is a unitless scaling factor of order unity. Here $\nu$ is the frequency of observed radiation in gigahertz, $T_{e}$ is the electron gas temperature in kelvins, $S_{\nu}$ is observed radio flux density in janskys, and $D$ is the distance to the cloud from the observer measured in kiloparsecs. We assume an average $\mathrm{H}$ II temperature of $T_{e}=10^{4} \mathrm{~K}$. The results of these calculations are listed in Table 3.
Similar to our findings in M83 (Maddox et al. 2006), we detect only the brightest $\mathrm{H}$ II regions in our observations. The larger sample of optically fainter $\mathrm{H}$ II regions are below the detection threshold of our radio observations. Eleven of the sources exhibit steep spectral indices $(\alpha<-0.3)$, indicating a nonthermal emission mechanism. Five of the source are confirmed SNRs (see $\S 4.1 .3$ ), and the remaining ones are SNR candidates. Highresolution $[\mathrm{O}$ III] and $[\mathrm{S}$ II] observations are needed to confirm the classification of these sources as SNRs.

\subsubsection{X-Ray Counterparts}

Many radio sources also coincide with bright $\mathrm{X}$-ray sources in external galaxies. Although most of the luminous X-ray sources detected in galaxies are XRBs, few are detectable in the radio, assuming isotropic emission. Radio emission from these sources is typically very weak in the long periods of quiescence. It may, however, be possible to detect beamed emission, e.g., from a microquasar. The presence of such an X-ray counterpart can aid in the classification of these radio sources.

Of the 107 radio sources detected in M51, 13 have X-ray counterparts from Chandra observations (Kilgard et al. 2005). The Chandra observations reach a uniform limiting luminosity of less than $5 \times 10^{36} \mathrm{ergs} \mathrm{s}^{-1}$. Basic properties of these 13 sources are listed in Table 4. As described in Prestwich et al. (2003) and Kilgard et al. (2005), it is possible to assign a rough source classification 
TABLE 3

Excitation Parameters for $\mathrm{H} \alpha$ Sources

\begin{tabular}{|c|c|c|}
\hline Source & Spectral Index & $\begin{array}{c}U \\
(\mathrm{pc} \mathrm{cm}-2)\end{array}$ \\
\hline $5 \ldots$ & $>+0.09$ & 242.5 \\
\hline 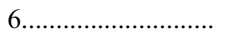 & $-0.46 \pm 0.55$ & 184.2 \\
\hline 7............................ & $0.05 \pm 0.09$ & 369.9 \\
\hline 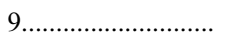 & $0.14 \pm 0.12$ & 355.0 \\
\hline $10 \ldots \ldots \ldots \ldots \ldots \ldots \ldots$ & $0.25 \pm 0.43$ & 244.7 \\
\hline 11 .......................... & $>-0.06$ & 228.4 \\
\hline 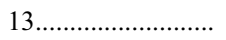 & $>+0.98$ & 348.7 \\
\hline 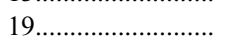 & $-0.59 \pm 0.13$ & 280.5 \\
\hline $22 \ldots \ldots \ldots \ldots \ldots \ldots \ldots \ldots \ldots \ldots \ldots \ldots \ldots \ldots$ & $-0.49 \pm 0.25$ & 225.9 \\
\hline $24 \ldots \ldots \ldots \ldots \ldots \ldots \ldots$ & $-0.1 \pm 0.34$ & 230.9 \\
\hline 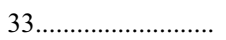 & $-0.09 \pm 0.3$ & 242.5 \\
\hline $42 \ldots \ldots \ldots \ldots \ldots \ldots \ldots \ldots \ldots \ldots \ldots$ & $-0.65 \pm 0.27$ & 216.6 \\
\hline 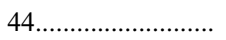 & $0.11 \pm 0.15$ & 323.3 \\
\hline $46 \ldots \ldots \ldots \ldots \ldots \ldots \ldots$ & $-0.03 \pm 0.36$ & 229.7 \\
\hline 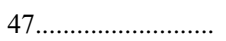 & $-0.6 \pm 0.41$ & 198.5 \\
\hline 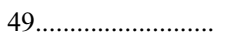 & $-0.45 \pm 0.42$ & 213.8 \\
\hline 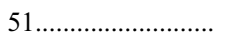 & $>-0.13$ & 222.0 \\
\hline 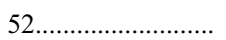 & $-0.82 \pm 0.27$ & 225.9 \\
\hline 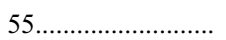 & $-0.3 \pm 0.28$ & 233.3 \\
\hline 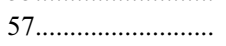 & $>-0.06$ & 228.4 \\
\hline 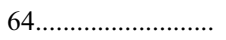 & $-0.5 \pm 0.2$ & 241.4 \\
\hline 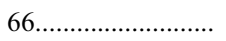 & $0.03 \pm 0.39$ & 229.7 \\
\hline 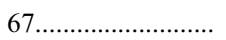 & $-0.28 \pm 0.19$ & 259.1 \\
\hline 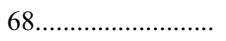 & $-0.78 \pm 0.27$ & 228.4 \\
\hline 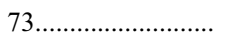 & $-0.19 \pm 0.3$ & 250.0 \\
\hline 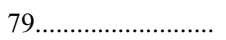 & $-0.26 \pm 0.1$ & 355.5 \\
\hline $82 \ldots \ldots \ldots \ldots \ldots \ldots \ldots \ldots$ & $0.01 \pm 0.34$ & 247.9 \\
\hline 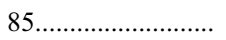 & $-0.08 \pm 0.27$ & 259.1 \\
\hline 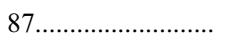 & $-0.19 \pm 0.02$ & 613.2 \\
\hline 89 & $-0.04 \pm 0.27$ & 254.1 \\
\hline 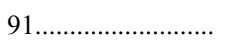 & $0.04 \pm 0.39$ & 234.5 \\
\hline 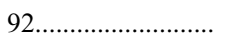 & $-0.06 \pm 0.31$ & 245.8 \\
\hline 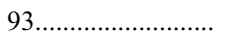 & $>-0.10$ & 224.6 \\
\hline 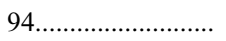 & $0.15 \pm 0.34$ & 242.5 \\
\hline 97........................ & $0.06 \pm 0.06$ & 442.3 \\
\hline 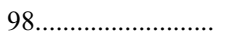 & $0.09 \pm 0.45$ & 227.2 \\
\hline 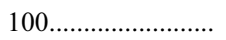 & $0.11 \pm 0.14$ & 335.9 \\
\hline 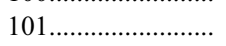 & $-0.04 \pm 0.37$ & 232.1 \\
\hline 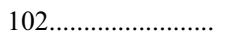 & $>+0.14$ & 247.9 \\
\hline 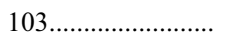 & $0.16 \pm 0.23$ & 304.1 \\
\hline $105 \ldots \ldots \ldots \ldots \ldots$ & $0.16 \pm 0.17$ & 324.6 \\
\hline
\end{tabular}

to an X-ray source based on its X-ray luminosity, variability, and placement on an X-ray color-color diagram. Figure 7 shows a model X-ray color-color diagram. SNRs, which typically have thermal X-ray spectra with temperatures of a few hundred electron volts, are located in the lower-left portion of the color-color diagram. As the spectrum becomes more absorbed, the source moves up in the diagram. The right half of the diagram is occupied by sources with power-law or multicomponent spectra. Thus, all SNRs should be found on the left side of the diagram, regardless of the absorbing column densities.

Figure 7 shows the X-ray color-color diagram for the radio sources with X-ray counterparts. As can clearly be seen, most of the sources have X-ray colors indicative of thermal spectra with increasing absorption. Indeed, 8 of the 13 sources are near-textbook examples of SNR-like X-ray spectra. These sources are numbers $47,49,52,58,68,73,79$, and 84 .

Of the eight sources with SNR-like X-ray colors, six are coincident with $\mathrm{H} \alpha$ shells or discrete $\mathrm{H} \alpha$ point sources in observations with the ACS on HST (optical/X-ray coincidences are discussed in Kilgard et al. 2006). The remaining two sources, 73 and 79, are associated with massive star clusters with embedded $\mathrm{H} \alpha$ emission; thus, determining the position of any individual SNRs in the $\mathrm{H} \alpha$ is not feasible. These sources are located along the spiral arms of the galaxy. Source 58, along the inner spiral arm, is in a confused $\mathrm{H} \alpha$ emission region, although we have already determined it to be emission from SN 1994I.

A brief discussion of each of the remaining five radio sources with X-ray counterparts, which do not exhibit SNR-like X-ray properties, is provided here:

1. Source 4 lies outside the main disk of the galaxy. Its X-ray colors are consistent with an XRB-like spectrum, although a soft active galactic nucleus (AGN) spectrum cannot be ruled out. The source is coincident with a blue point source in the optical ACS images.

2. Source 12 has a highly absorbed X-ray spectrum, with no photons detected below $2 \mathrm{keV}$ in any of the Chandra observations. (It is located at the far right of the color-color diagram.) It also exhibits variability on a timescale of months to years between Chandra observations. The source is coincident with a very faint red point source in the optical images from ACS. The steep radio spectrum points to a highly nonthermal emission mechanism. All these things combined lead to the conclusion that the source is likely a background AGN whose soft X-ray emission is absorbed by M51.

3. Source 65 is coincident with a compact $\mathrm{H} \alpha$ source, but exhibits XRB-like X-ray colors.

4. Source 95 is highly absorbed in the X-rays (it is located in the "absorbed sources" ellipse of the color-color diagram). It is thus impossible to decide between XRB or SNR spectra. However, the position toward the left on the color-color diagram means that we can rule out hard X-ray spectra. The source is thus consistent with having either an SNR-like or soft XRB-like spectrum. There is no counterpart detected in $B, V, I$, or $\mathrm{H} \alpha$.

5. Source 107 is the brightest of the X-ray/radio overlaps. It exhibits a flat radio spectrum between 20 and $6 \mathrm{~cm}$. This would normally indicate a thermal process that would also produce $\mathrm{H} \alpha$ emission; however, no $\mathrm{H} \alpha$ emission is detected in the ACS images. The radio observations were separated by $\sim 20$ months. It is possible that we are observing a microquasar in a radio-loud state (Nipoti et al. 2005).

\subsection{Nuclear Emission}

The X-ray and radio morphologies of the nuclear region are strikingly similar. They consist of an area of ringlike emission to the north, postulated to be a bubble blown out due to an outflow (Ford et al. 1985), and a dense area of emission to the south of the nucleus. Following the studies by Terashima \& Wilson (2001, and references therein), we refer to the southern source as the extranuclear cloud (XNC). The nucleus itself is unresolved. Figure 8 shows radio contours overlaid on a three-color X-ray image of this nuclear region.

X-ray spectral modeling of the nucleus determined that the emission consisted of two major components. The hard component arises from reflection of the radiation from a compact nuclear source with a power-law spectrum by cold matter in the vicinity. The soft component is similar to the XNC, being well modeled by a thermal plasma that is shock-heated by mass outflow (Terashima $\&$ Wilson 2001). The shock-heating process results in nonthermal continuum radio emission, as shown in Crane \& van der Hulst (1992). For the nucleus (source 52), we measure a steep spectral index $(-0.82 \pm 0.27)$. 
TABLE 4

X-Ray Counterparts to Radio Sources

\begin{tabular}{|c|c|c|c|c|c|c|}
\hline \multirow[b]{2}{*}{$\begin{array}{l}\text { SOURCE } \\
\text { (1) }\end{array}$} & \multicolumn{2}{|c|}{ Flux Density } & \multirow[b]{2}{*}{$\begin{array}{c}\text { Spectral Index }{ }^{\mathrm{a}} \\
\text { (4) }\end{array}$} & \multirow[b]{2}{*}{$\begin{array}{l}\text { CXOU } \\
(5)\end{array}$} & \multirow{2}{*}{$\begin{array}{c}L_{\mathrm{X}}(0.3-2 \mathrm{keV}) \\
\left(10^{37} \mathrm{ergs} \mathrm{s}^{-1}\right) \\
(6)\end{array}$} & \multirow[b]{2}{*}{$\begin{array}{c}\text { X-RAY ID } \\
\text { (7) }\end{array}$} \\
\hline & $\begin{array}{l}20 \mathrm{~cm} \\
\text { (2) }\end{array}$ & $\begin{array}{c}6 \mathrm{~cm} \\
(3)\end{array}$ & & & & \\
\hline 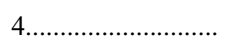 & $84 \pm 22$ & $102 \pm 18$ & +0.15 & $\mathrm{~J} 132939.0+471324$ & 5 & $\mathrm{XRB} / \mathrm{SNR}$ \\
\hline 12 & $123 \pm 21$ & $43 \pm 15$ & -0.86 & $\mathrm{~J} 132949.1+471257$ & 3 & ABS \\
\hline $47 \ldots$ & $84 \pm 21$ & $40 \pm 13$ & -0.60 & $\mathrm{~J} 132952.1+471127$ & 6 & SNR \\
\hline $49 \ldots \ldots \ldots \ldots \ldots$ & $87 \pm 29$ & $50 \pm 13$ & -0.45 & $\mathrm{~J} 132952.2+471129$ & 4 & SNR \\
\hline ................... & $162 \pm 26$ & $59 \pm 13$ & -0.82 & $\mathrm{~J} 132952.7+471121$ & 3 & SNR \\
\hline $58^{\mathrm{a}} \ldots \ldots \ldots \ldots \ldots \ldots$ & $162 \pm 26$ & $93 \pm 14$ & -0.45 & $\mathrm{~J} 132954.2+471130$ & 4 & SNR \\
\hline $65 \ldots \ldots \ldots \ldots \ldots \ldots \ldots \ldots \ldots \ldots \ldots \ldots$ & $406 \pm 21$ & $280 \pm 17$ & -0.30 & $\mathrm{~J} 132955.0+470922$ & 7 & $\mathrm{XRB} / \mathrm{SNR}$ \\
\hline 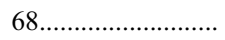 & $158 \pm 23$ & $61 \pm 14$ & -0.78 & $\mathrm{~J} 132955.2+471046$ & 1 & SNR \\
\hline $73 \ldots \ldots \ldots \ldots \ldots \ldots \ldots \ldots$ & $101 \pm 23$ & $80 \pm 16$ & -0.19 & $\mathrm{~J} 132955.4+471402$ & 2 & SNR \\
\hline $79 \ldots \ldots \ldots \ldots \ldots \ldots \ldots \ldots$ & $319 \pm 25$ & $230 \pm 13$ & -0.26 & $\mathrm{~J} 132955.9+471144$ & 7 & SNR \\
\hline $84 \ldots \ldots \ldots \ldots \ldots \ldots$ & $128 \pm 22$ & $59 \pm 14$ & -0.63 & $\mathrm{~J} 132957.5+471037$ & 3 & SNR \\
\hline 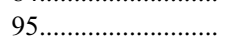 & $131 \pm 22$ & $62 \pm 15$ & -0.61 & $\mathrm{~J} 133001.3+471244$ & 2 & ABS \\
\hline 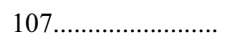 & $500 \pm 22$ & $482 \pm 20$ & -0.03 & $\mathrm{~J} 133011.0+471041$ & 1 & XRB \\
\hline
\end{tabular}

Notes.-Col. (1): Radio source number from Table 1. Cols. (2) and (3): Radio flux densities. Col. (4): Radio spectral index derived from flux densities. Col. (5): Chandra source designation. Col. (6): 0.3-2 keV X-ray luminosity of associated X-ray source. Col. (7): Source classification based on X-ray colors.

${ }^{a}$ Coincident with SN 1994I.

We measure a similar steep spectrum for the XNC $(\alpha=$ $-0.81 \pm 0.02)$. In high-resolution $6 \mathrm{~cm}$ observations of the nucleus, Crane \& van der Hulst (1992) identify a radio jet emanating from the nucleus and terminating in the XNC. The jet drives the heating of the cloud and fuels the expansion of the $\mathrm{XNC}$, as suggested by, e.g., Ford et al. (1985). The jet is the site of the hardest X-ray emission in the XNC (Terashima \& Wilson 2001) and the broadest optical line emission (Cecil 1988). Gopal-Krishna \& Irwin (2000) presented a model in which shells in spiral galaxies, like those seen in our data, may be produced through shock heating due to a radio jet. The interaction of the jet with the ma- terial within the disk would lead to a bow shock, which is seen in the XNC by Crane \& van der Hulst (1992). Our radio observations lack the resolution of Crane \& van der Hulst (1992) and are unable to resolve the radio jet.

Comparison of the bubble in the two radio bands yields a nonthermal spectral index measurement ( $\alpha=-0.58)$, which is consistent with a synchrotron emission mechanism. This matches the X-ray analysis of the bubble in Terashima \& Wilson (2001). The knots of X-ray emission (not seen in the our radio observations due to resolution limits) may be SNRs. These sources are not heavily absorbed, so they could also be clumps of hot gas on the
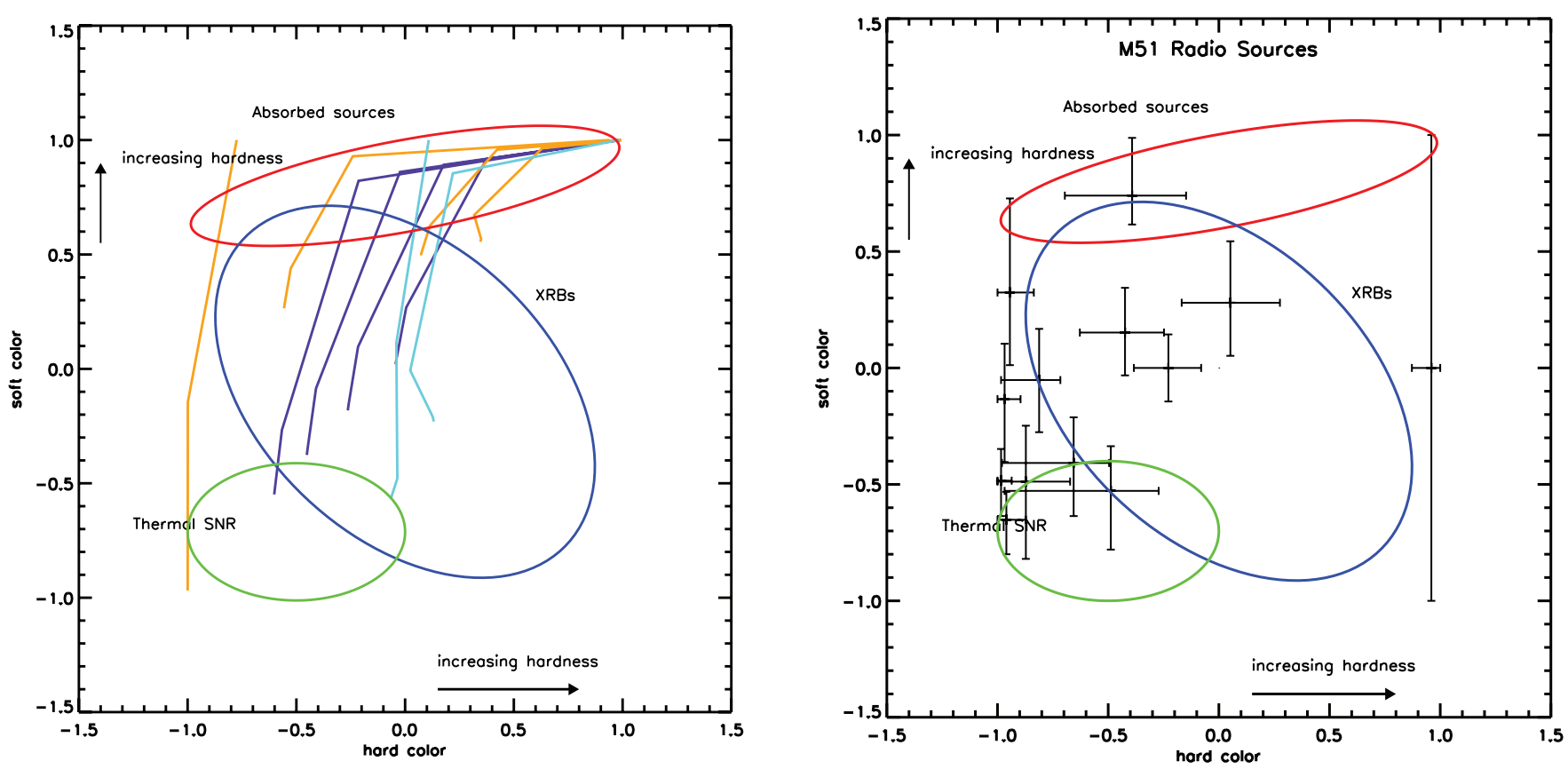

FIG. 7.-Left: Model X-ray color-color diagram. The purple lines are power-law spectra with photon index increasing from left to right $(\Gamma=1,1.5,2$, and 2.5$)$ and absorption increasing from bottom to top $\left(0-10^{24} \mathrm{~cm}^{-2}\right)$. The orange lines are thermal spectra with temperature increasing from left to right $(0.5,1,1.5$, and $2 \mathrm{keV})$ and absorption increasing with the power-law spectra. The light-blue lines are power-law plus blackbody spectra. The ellipses mark the likely location of thermal SNRs, XRBs, and sources with very heavily absorbed spectra (where color classification becomes difficult). Right: X-ray color-color diagram following Kilgard et al. (2005) for our radio sources with X-ray counterparts. 


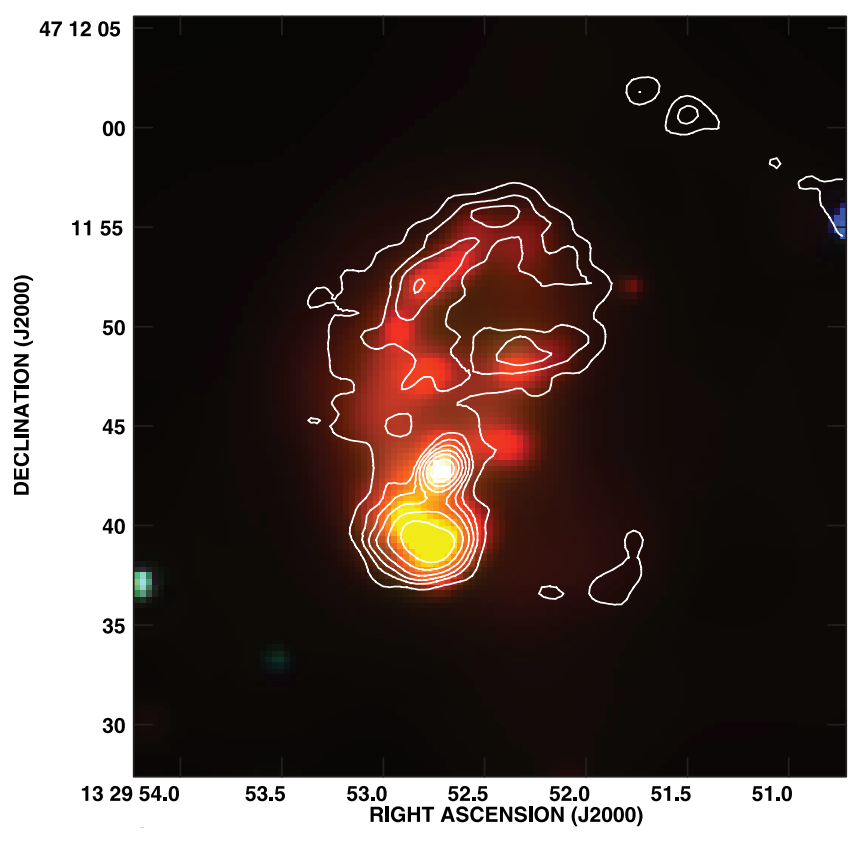

FIG. 8.-Enlargement of the nuclear region of M51. The colors represent $\mathrm{X}$-ray emission, while the contours represent $6 \mathrm{~cm}$ radio emission. The smallest pointlike feature in each image is $\sim 1.0^{\prime \prime}$. The radio contour levels are 70, 98, 200, $280,400,560$, and $800 \mu \mathrm{Jy}_{\text {beam }^{-1}}$.

edge of the bubble or soft XRBs that are not embedded in the diffuse emitting gas. Our observations and those of Crane \& van der Hulst (1992) indicate no direct evidence for jet emission in the bubble region of the nucleus. The ringlike structure of the bubble indicates cooler gas within, which along with a shallower spectral index make it unlikely that the bubble is powered by a continuous jet. Rudnick (1982) suggested a one-sided ejection mechanism to explain the asymmetrical nature of some doublelobed radio sources. If this is the case, the bubble could be a relic of a previous one-sided ejection cycle by the AGN of M51a.

\section{CONCLUSIONS}

We have presented a multiwavelength study of the compact radio sources in M51. Primarily using radio, optical, and X-ray observations, we have classified most of the detected radio sources. Our primary findings are as follows:

1. We detected 107 compact radio sources, 44 with large H II counterparts, and 24 additional sources that are associated with stellar clusters.

2. Thirteen radio sources have X-ray counterparts. Eight of the 13 sources have SNR-like X-ray spectra. Two of these X-ray/ radio sources are coincident with resolved $\mathrm{H} \alpha$ shells. Two of the remaining sources are highly absorbed. Source 12 is likely a background AGN, while source 95, although there is no optical counterpart, could be a dust-embedded SNR or an XRB.

3. Six of the radio sources associated with $\mathrm{H} \alpha$ sources are young SNRs with resolved shells. Most exhibit steep-continuum radio spectra consistent with synchrotron emission. Based on a Cas A expansion velocity, we estimate the age of the SNRs to be $\sim 2000-3300 \mathrm{yr}$.

4. We detected emission from the Type Ic SN 1994I nearly a decade after explosion. We found the emission at this epoch to be consistent with light-curve models for Type Ib/Ic SNe. The SN was also detected in X-ray, although no optical counterpart was seen.

5. The result of our analysis of the nuclear region, including the XNC, bubble, and nucleus, are consistent with previous studies that suggest a bipolar radio jet from the low-luminosity Seyfert 2 nucleus. The nucleus and XNC have identical continuum spectra, indicative of shock-heated gas interacting with the jet.

This work employed extensive use of the NASA Extragalactic Database. This work was supported by NSF grant AST 0307279 (J. J. C.). C. J. S. is a Cottrell Scholar of Research Corporation, and work on this project has been supported by the NASA Wisconsin Space Grant Consortium. We wish to thank the anonymous referee for very useful comments that have improved the quality of this manuscript.

\section{REFERENCES}

Baron, E., Hauschildt, P. H., Branch, D., Kirshner, R. P., \& Filippenko, A. V.

Nipoti, C., Blundell, K. M., \& Binney, J. 2005, MNRAS, 361, 633 1996, MNRAS, 279, 799

Calzetti, D., et al. 2005, ApJ, 633, 871

Cecil, G. 1988, ApJ, 329, 38

Crane, P. C., \& van der Hulst, J. M. 1992, AJ, 103, 1146

Feldmeier, J. J., Ciardullo, R., \& Jacoby, G. H. 1997, ApJ, 479, 231

Ford, H. C., Crane, P. C., Jacoby, G. H., Lawrie, D. G., \& van der Hulst, J. M. 1985, ApJ, 293, 132

Gopal-Krishna, \& Irwin, J. A. 2000, A\&A, 361, 888

Immler, S., Wilson, A. S., \& Terashima, Y. 2002, ApJ, 573, L27

Kilgard, R. E., Prestwich, A. H., Ward, M. J., \& Roberts, T. P. 2006, in IAU Symp. 230, Populations of High-Energy Sources in Galaxies, ed. E. J. A.

Meurs \& G. Fabbiano (Cambridge: Cambridge Univ. Press), 189

Kilgard, R. E., et al. 2005, ApJS, 159, 214

Kloehr, W., Muendlein, R., Li, W., Yamaoka, H., \& Itagaki, K. 2005, IAU Circ. 8553

Kowal, C. T., \& Sargent, W. L. W. 1971, AJ, 76, 756

Maddox, L. A., Cowan, J. J., Kilgard, R. E., Lacey, C. K., Prestwich, A. H., Stockdale, C. J., \& Wolfing, E. 2006, AJ, 132, 310 (Paper I)

Mezger, P. G., \& Henderson, A. P. 1967, ApJ, 147, 471

Prestwich, A. H., Irwin, J. A., Kilgard, R. E., Krauss, M. I., Zezas, A., Primini,

F., Kaaret, P., \& Boroson, B. 2003, ApJ, 595, 719

Puckett, T., et al. 1994, IAU Circ. 5961

Rots, A. H., Bosma, A., van der Hulst, J. M., Athanassoula, E., \& Crane, P. C. 1990, AJ, 100, 387

Rudnick, L. 1982, in IAU Symp. 97, Extragalactic Radio Sources, ed. D. S. Heeschen \& C. M. Wade (Dordrecht: Reidel ), 47

Sandage, A., \& Tammann, G. A. 1974, ApJ, 194, 559

Schraml, J., \& Mezger, P. G. 1969, ApJ, 156, 269

Stockdale, C. J., Kelley, M., van Dyk, S. D., Sramek, R. A., Weiler, K. W., \& Panagia, N. 2005a, IAU Circ. 8603

Stockdale, C. J., Sramek, R. A., Weiler, K. W., Van Dyke, S. D., Pangia, N., Montes, M. J., \& Rupen, M. P. 2005b, Poster at IAU Colloq. 192, Cosmic Explosions on the 10th Anniversary of SN1993J, ed. J.-M. Marcaide \& K. W. Weiler (Berlin: Springer)

Terashima, Y., \& Wilson, A. S. 2001, ApJ, 560, 139

Tingay, S. J. 2004, AJ, 127, 10

Weiler, K. W., Panagia, N., Montes, M. J., \& Sramek, R. A. 2002, ARA\&A, 40, 387 